$\sqrt{6}$

EDITOR'S

CHOICE

\title{
Disorders of iron metabolism. Part 1: molecular basis of iron homoeostasis
}

\author{
Manuel Muñoz, ${ }^{1}$ José Antonio García-Erce, ${ }^{2}$ Ángel Francisco Remacha ${ }^{3}$
}

${ }^{1}$ Transfusion Medicine, School of Medicine, University of Málaga, Málaga, Spain 'Hematology and Hemotherapy, University Hospital Miguel Servet, Zaragoza, Spain ${ }^{3}$ Hematology and Hemotherapy, Complejo Hospitalario de Toledo, Toledo, Spain

\section{Correspondence to} Professor Manuel Muñoz, Medicina Transfusional Facultad de Medicina Campus de Teatinos, s/n 29071-Málaga, Spain; mmunoz@uma.es

All author contributed equally to the design, writing and discussion of this paper.

Accepted 15 November 2010 Published Online First 20 December 2010
ABSTRACT

Iron functions Iron is an essential micronutrient, as it is required for satisfactory erythropoietic function, oxidative metabolism and cellular immune response.

Iron physiology Absorption of dietary iron (1-2 mg/

day) is tightly regulated and just balanced against iron loss because there are no active iron excretory mechanisms. Dietary iron is found in haem (10\%) and non-haem (ionic, 90\%) forms, and their absorption occurs at the apical surface of duodenal enterocytes via different mechanisms. Iron is exported by ferroportin 1 (the only putative iron exporter) across the basolateral membrane of the enterocyte into the circulation (absorbed iron), where it binds to transferrin and is transported to sites of use and storage. Transferrinbound iron enters target cells-mainly erythroid cells, but also immune and hepatic cells — via receptormediated endocytosis. Senescent erythrocytes are phagocytosed by reticuloendothelial system macrophages, haem is metabolised by haem oxygenase, and the released iron is stored as ferritin. Iron will be later exported from macrophages to transferrin. This internal turnover of iron is essential to meet the requirements of erythropoiesis (20-30 mg/day). As transferrin becomes saturated in iron-overload states, excess iron is transported to the liver, the other main storage organ for iron, carrying the risk of free radical formation and tissue damage.

Regulation of iron homoeostasis Hepcidin, synthesised by hepatocytes in response to iron concentrations, inflammation, hypoxia and erythropoiesis, is the main iron-regulatory hormone. It binds ferroportin on enterocytes, macrophages and hepatocytes triggering its internalisation and lysosomal degradation. Inappropriate hepcidin secretion may lead to either iron deficiency or iron overload.

\section{INTRODUCTION}

Iron is an essential micronutrient, as it is required for satisfactory erythropoietic function, oxidative metabolism and cellular immune response. For a $70 \mathrm{~kg}$ man, total body iron is about $3500 \mathrm{mg}$ (50 mg/kg body weight). Most of the iron in the body is distributed in red blood cell haemoglobin (65\%; $2.300 \mathrm{mg}$ ). Approximately $10 \%$ is found in muscle fibres (in myoglobin) and other tissues (in enzymes and cytochromes) (350 mg). The remaining body iron is stored in the liver $(200 \mathrm{mg}$ ), macrophages of the reticuloendothelial system (RES) (500 mg) and bone marrow (150 mg).

On the other hand, the body has no active means of excreting iron, and thus regulation of absorption of dietary iron from the duodenum plays a critical role in iron homoeostasis. ${ }^{1}$ This is extremely important as iron is essential for cellular metabolism and aerobic respiration, and cellular iron overload leads to toxicity and cell death via free radical formation and lipid peroxidation. Thus, iron homoeostasis requires tight regulation. ${ }^{2-4}$

In this paper, we will review the main pathways of iron metabolism and their regulation (part I), whereas the causes of iron deficiency and iron overload, and the different laboratory tests to establish a correct diagnosis of iron overload, iron deficiency and anaemia, and the indications, advantages and side effects of the different options for treating iron overload and iron deficiency will be discussed in the second paper (part II)

\section{MAIN PATHWAYS OF IRON HOMOEOSTASIS Iron absorption}

The normal Western diet contains 15-20 mg iron in haem (10\%) and non-haem (ionic, $90 \%$ ) forms, of which 1-2 $\mathrm{mg}$ are absorbed daily mostly at the duodenum. Iron absorption is balanced against iron loss through sloughed intestinal mucosal cells, menstruation and other blood losses. Daily iron absorption may increase in response to increased iron demand (eg, growth, pregnancy or blood loss).

Dietary non-haem iron primarily exists in an oxidised $\left(\mathrm{Fe}^{3+}\right)$ form that is not bioavailable and must first be reduced to the $\mathrm{Fe}^{2+}$ form by a ferrireductase enzyme, which uses vitamin $C$ as coenzyme, before being transported across the intestinal epithelium. This is accomplished by a carrier protein called divalent metal transporter 1 (DMT1), which also traffics other metal ions such as zinc, copper and cobalt by a proton-coupled mechanism. ${ }^{3}{ }^{4}$ The absorption of non-haem iron can be diminished by co-administration of tretracyclines, proton pump inhibitor and antacid medication, phytates (high-fibre diets), calcium and phenolic compounds (coffee, tea). In addition, Helicobater pylori infection produces gastric atrophy, which can lead to profound iron-deficiency anaemia. ${ }^{5}$

Haem iron is absorbed into the enterocyte by a putative, not completely identified, haem carrier protein $1 .{ }^{6}$ Once internalised in the enterocyte, it is likely that most dietary haem is metabolised by haem oxygenase to release $\mathrm{Fe}^{2+}$, which enters a common pathway with dietary non-haem iron before it leaves the enterocyte ${ }^{2}{ }^{3}$ (figure 1). However, it remains uncertain whether some intact haem traverses the cell, leaving the enterocyte through the action of the recently characterised haem exporters, which are also expressed in kidney, liver and erythroblast, suggesting that they may act at those sites. ${ }^{7}$ Plasma haem is scavenged and transported by haemopexin to hepatocytes for degradation. 


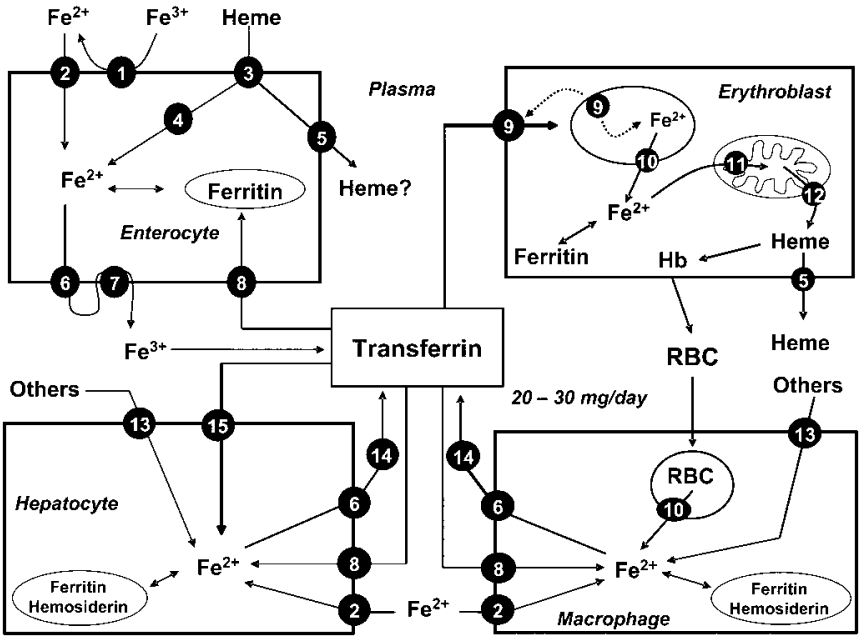

Figure 1 Main pathways of iron metabolism in mammals (modified from Muñoz et $a l^{2}$ ). Key: 1, ferrireductase; 2, divalent metal transporter (DMT1); 3, haem protein carrier 1 (HPC1); 4, haem oxygenase; 5 , haem exporter; 6, ferroportin (Ireg-1); 7, hephaestin/caeruloplasmin; 8, transferrin receptor-1 (TfR1); 9, diferric transferring-TfR1 complex; 10, natural resistance macrophage protein (Nramp-1); 11, mitoferrin; 12, mitochondrial haem exporter $(A b c b 6) ; 13$, others: bacteria, lactoferrin, haemoglobin-haptoglobin, haem-haemopexin, etc; 14, caeruloplasmin; 15, transferrin receptor-2 (TfR2). See text for further details.

Once inside the intestinal epithelial cell, most $\mathrm{Fe}^{2+}$ is exported by ferroportin 1 across the basolateral membrane of the enterocyte (absorbed iron), and oxidised to $\mathrm{Fe}^{3+}$ by hephaestin before being bound by plasma transferrin. Ferroportin 1 is also expressed in hepatocytes, reticuloendothelial macrophages and placental syncytiotrophoblasts (where it regulates iron entry into fetal circulation). ${ }^{8}$

\section{Iron distribution}

Iron released into the circulation binds to transferrin and is transported to sites of use and storage. About $30-40 \%$ of the iron-binding capacity of transferrin is used in normal physiological conditions; thus transferrin-bound iron is $\sim 4 \mathrm{mg}$, but this is the most important dynamic iron pool. ${ }^{9}$ Transferrinbound iron enters target cells-mainly erythroid cells, but also immune and hepatic cells - through a highly specific process of receptor-mediated endocytosis (figure 1). As diferric transferrin binds to transferrin receptor 1 (TfR1) at the plasma membrane, patches of cell-surface membrane carrying receptor-ligand complexes invaginate to form clathrin-coated endosomes (siderosomes). ${ }^{9}$ After clathrin is removed, the siderosomes become acidified through ATP-dependent proton influx, mediated by a family of STEAP (six transmembrane epithelial antigen of the prostate) proteins, leading to conformational changes in both transferrin and TfR1, and promoting the release of $\mathrm{Fe}^{3+}$ from transferrin. $\mathrm{Fe}^{3+}$ is then reduced to $\mathrm{Fe}^{2+}$ by a ferrireductase and transported to the cytoplasm through the DMT1, whereas TfR1 is recycled to the cell membrane and transferrin is shed back into the circulation $^{2} 10$ (figure 1). Within the erythroblast, the mitochondrial iron importer, mitoferrin, plays a critical role in supplying iron to ferrochelatase for insertion into protoporphyrin IX to form haem (the final step of mitochondrial haem synthesis) ${ }^{10}$ (figure 1). There is some evidence that, in developing erythroid cells, iron could be delivered directly from the siderosomes into the mitochondria. ${ }^{11}$ Finally, haem exporters transfer haem from the mitochondria to the cytosol and remove excess haem from the erythroid cells (figure 1).

\section{Iron storage}

Haemoglobin iron has a substantial turnover, as senescent erythrocytes are phagocytosed by RES macrophages. Within the phagocytic vesicles, haem is metabolised by haem oxygenase, and the released $\mathrm{Fe}^{2+}$ is exported to the cytoplasm through the action of NRAMP1 (natural resistance-associated macrophage protein-1), a transport protein similar to DMT1 (figure 1). Macrophages can also obtain iron from bacteria and apoptotic cells, from plasma through the action of DMT1 and TfR1, and from other sources (figure 1). In the cell, iron can be stored in two forms: in the cytosol as ferritin, and, after breakdown of ferritin, in the lysosomes as haemosiderin. Haemosiderin represents a very small fraction of normal body iron stores, mostly in macrophages, but increases dramatically during iron overload. ${ }^{9}$ Importantly, iron storage in the macrophages is safe, as it does not lead to oxidative damage.

Iron export from macrophages to transferrin is accomplished primarily by ferroportin 1, the same iron-export protein as expressed in the duodenal enterocyte, and caeruloplasmin ${ }^{2}$ (figure 1). The amount of iron required for daily production of 300 billion red blood cells $(20-30 \mathrm{mg}$ ) is provided mostly by macrophage iron recycling. ${ }^{3}$ Therefore, as daily absorption (1-2 mg) just balances daily loss, internal turnover of iron is essential to meet the bone marrow requirements for erythropoiesis. $^{1-3}$

The liver is the other main storage organ for iron, and the generation of free radicals and lipid peroxidation products in iron-overload states may result in progressive hepatic tissue injury and eventually cirrhosis or hepatocellular carcinoma. ${ }^{12}$ The uptake of transferrin-bound iron by the liver from plasma is mediated by TfR1 and TfR2 (figure 1), although it can acquire iron from non-transferrin-bound iron (by a carrier-mediated process consistent with DMT1), ferritin, haemoglobin-haptoglobin complexes and haem-haemopexin complexes. Iron is sequestrated in hepatocytes predominantly in the form of ferritin or haemosiderin. In contrast, once again, ferroportin 1 is likely to be the only protein mediating the export of iron from hepatocytes, which is then oxidised by caeruloplasmin and bound to transferrin ${ }^{2}$ (figure 1).

Iron storage in cardiomyocytes is also of great interest, as cardiac failure is the leading cause of death among patients with untreated hereditary haemochromatosis or transfusionassociated iron overload. ${ }^{2}$ In cardiac cells, excess iron may result in oxidative stress and alteration of myocardial function due to DNA damage by hydrogen peroxide through the Fenton reaction. ${ }^{2}$

\section{REGULATION OF IRON HOMOEOSTASIS}

The absorption of iron by duodenal enterocytes is dependent on body iron stores, hypoxia, inflammation and rate of erythropoiesis. Two regulatory models have been proposed, both of which may contribute to the regulation of iron absorption: the crypt programming model and the hepcidin model. The crypt programming model proposes that enterocytes in the crypts of the duodenum take up iron from the plasma, via TfR1 and TfR2. The intracellular iron concentration controls the interaction of cytosolic iron regulatory proteins (IRPs) 1 and 2 with ironresponsive elements (IREs). In the absence of iron, IRP1 binds to IREs of TfR1, DMT1 and ferroportin 1 mRNA, the transcript is stabilised, translation proceeds, and the proteins are synthesised. Thus, a high IRP-binding activity reflects low body iron stores and results in upregulation of these proteins in the duodenum increasing dietary iron absorption. When IRPs bind to IREs 
of ferritin mRNA, translation of the transcript is blocked and synthesis is halted. Thus, ferritin concentrations are reciprocally regulated, being increased in iron-replete states and decreased in iron-deplete states. ${ }^{2}$

The hepcidin model proposes that hepcidin is produced mainly by hepatocytes in response to the iron content of the blood. Then, hepcidin is secreted into the bloodstream and interacts with villous enterocytes to regulate the rate of iron absorption by controlling the expression of ferroportin 1 at their basolateral membranes. The binding of hepcidin to ferroportin 1 initially causes Janus kinase 2-mediated tyrosine phosphorylation of the cytosolic loop of the carrier protein, phosphorylated ferroportin 1 is then internalised, dephosphorylated, ubiquitinated and ultimately degraded in the late endosome/lysosome compartment. Ferroportin 1 molecules present in macrophages and liver are also targets for hepcidin. ${ }^{13}$

Within normal iron concentration ranges, the sensing process probably involves the local iron-induced production of bone morphogenic proteins (BMPs) such as BMP6. Briefly, BMP6 binds hepatocyte cell surface BMP receptors (BMPRs) I and II together with the BMP coreceptor, haemojuvelin (HJV), initiating a signal that is conveyed intracellularly by phosphorylation of small mothers against decapentaplegic (Smad) proteins. Phosphorylated Smad1, Smad5 and Smad8 form a complex with the common mediator Smad4, before translocation to the nucleus and activating hepcidin expression ${ }^{14} 15$ (figure 2). The soluble form of HJV (sHJV), the release of which (HJV shedding) is inhibited by increasing extracellular concentrations of iron, is believed to compete for BMPR binding with its membraneanchored counterpart, thereby providing iron-sensitive modulation of hepcidin expression ${ }^{16}$ (figure 2). Iron may also stimulate the expression of other mediators and modulators, such as Smad6 and Smad7, which seem to attenuate the signal for hepcidin activation (figure 2).

The hepcidin regulatory signalling pathways may also be regulated by HFE. The HFE gene product (together with $\beta_{2}$ microglobulin) associates with both TfR1 and TfR2, but it is thought that the HFE-TfR2 complex senses transferrin saturation to modulate hepcidin transcription. ${ }^{17}$ In this model, diferric transferrin and HFE compete for binding to TfR1. In the presence of high concentrations of diferric transferrin, HFE is thought to dissociate from TfR1 and form part of an ironsensing complex through its interaction with TfR2. ${ }^{17} 18$ The interaction of transferrin-HFE with TfR2 may signal the high iron content of the blood to an iron sensor and signal-transduction effector complex, possibly the BMP-HJV complex or a distinct system. ${ }^{19}$ In this regard, binding of diferric transferrin to TfR2 inhibits HJV cleavage by furin, thus inhibiting the release of soluble HJV and resulting in cell-surface HJV-mediated enhanced responsiveness to BMPs and raised hepcidin concentration $^{20}$ (figure 2). Data from liver tissue of male patients with homozygote HFE mutations and significant iron overload showed appropriate induction of BMP6, but reduced Smad1/ Smad5/Smad8 phosphorylation relative to hepatic iron burden and failure of upregulation of hepcidin. Moreover, upregulation of Smad6 and Smad7 occurred in these patients, identifying these molecules as potential aggravators of disease pathogenesis $^{21}$ (figure 2).

There are also a number of factors that may downregulate liver hepcidin expression. Matriptase-2, a transmembrane serine protease encoded by the gene TMPRSS6, is predominantly expressed in liver, and has a critical role in iron homoeostasis. Matriptase-2 interacts with HJV and inhibits hepcidin synthesis by cleaving membrane HJV into fragments, which presumably

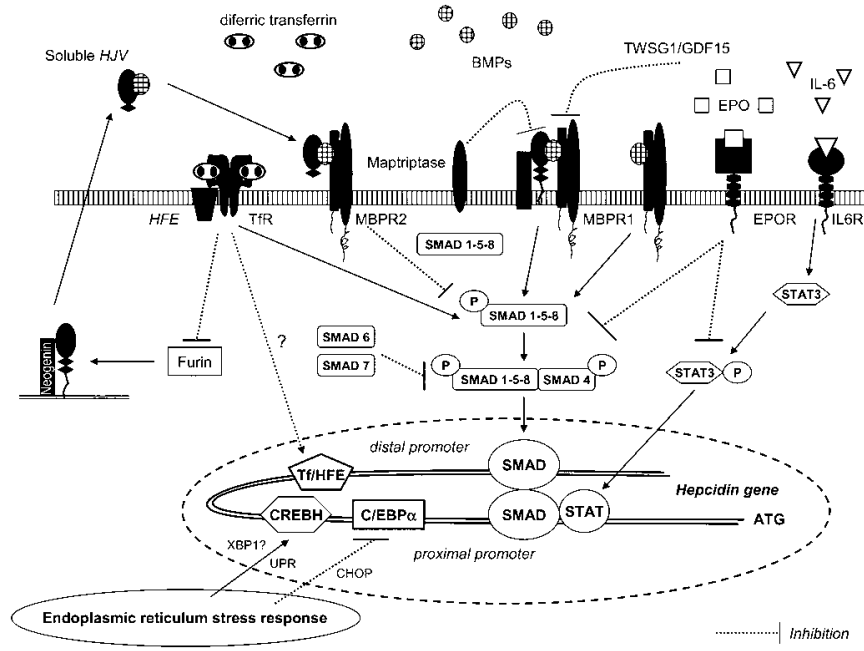

Figure 2 Multiple pathways for hepcidin regulation (adapted from references Pietrangelo ${ }^{19}$, Lee and Beutler ${ }^{20}$ and Wessling-Resnick ${ }^{45}$ ). $\mathrm{BMP}$, bone morphogenic protein; BMPR, BMP receptor; $\mathrm{C} / \mathrm{EBP} \alpha$, CCAAT/enhancer-binding protein; CHOP, CCAAT/enhancer-binding homologous protein (negative regulator of $\mathrm{C} / \mathrm{EBP} \alpha$ ); $\mathrm{CREBH}$, CAMPresponsive element binding protein $\mathrm{H}$; $\mathrm{EPO}$, erythropoietin; EPOR, EPO receptor; GDF15, growth differentiation factor 15; HFE, HFE gene product; HJV, haemojuvelin; IL-6, interleukin-6; IL6R, IL-6 receptor; STAT, signal transducer and activator of transcription; Tf, transferrin; TWSG1, twisted gastrulation; UPR, unfolded protein response; XBP-1, $\mathrm{X}$-box binding protein 1 (endoplasmic reticulum stress-activated transcription factor). See text for further details.

abolishes its function $^{22}$ (figure 2). Homozygous or compound heterozygous mutations in TMPRSS6 leading to hepcidin overexpression have been linked to familial iron-deficiency anaemia refractory to oral iron. ${ }^{23} 24$

It has been known for a long time that elevated erythropoiesis increases iron absorption regardless of body iron loading, and this process seems to be mediated by erythropoietin (EPO), growth differentiation factor 15 (GDF15) and twisted gastrulation (TWSG1). ${ }^{8}$ EPO suppression of hepcidin expression is mediated through the downregulation of (a) signal transducer and activator of transcription 3 (Stat3) phosphorylation triggered by lipopolysaccharides and (b) Smad1/5/8 phosphorylation induced by iron $^{25}$ (figure 2). A recent study showed that EPO administration in humans causes a marked and prolonged reduction in circulating hepcidin. ${ }^{26}$ In addition, there is evidence that EPO upregulates DMT1 and hephaestin gene expression in the duodenum ${ }^{27}$

GDF15 and TWSTG1 are members of the TGF- $\beta$ superfamily and are released under conditions of iron-restricted erythropoiesis. GDF15 is secreted by haemoglobinised erythroblasts during the final stages of erythropoiesis, which has been demonstrated to suppress hepcidin expression in vitro, ${ }^{28}$ whereas TWSTG1, which is produced mainly by the immature erythroid precursors during the early stages of erythropoiesis, suppresses the upregulation of hepcidin expression induced by $\mathrm{BMPs}^{29}$ (figure 2).

Hypoxia-inducible factors (HIFs) are multi-subunit transcription factors that are regulated by hydroxylation of an unstable $\alpha$ subunit controlling its degradation. This modification involves prolyl hydroxylases requiring both oxygen and iron; thus, under hypoxic or low-iron conditions, the prolyl hydroxylase activity is inhibited, resulting in the accumulation and translocation of HIF into the nucleus. HIF binding to the promoter of hepcidin leads to the suppression of hepcidin 
expression in hepatocytes, whereas its binding to the promoter of DMT1 increases DMT1 expression in enterocytes. ${ }^{30} 31$

In summary, it is hypothesised that, when hepcidin concentrations are increased in iron overload, iron release from intestinal crypt cells (and also from liver and macrophages) is reduced. Hepcidin concentrations are also increased during inflammation, regardless of the patient's iron status (see below). In contrast, when hepcidin concentrations are reduced, as in iron deficiency, anaemia or hypoxia, it is likely that ferroportin 1 expression and iron release from intestinal cells, liver and cells of the RES are increased. ${ }^{8}$ Genetic abnormalities in HFE, HJV, hepcidin and TfR2 lead to disproportionately low hepcidin concentrations and iron overload (haemochromatosis type I, IIa, IIb and III, respectively). In contrast, mutations in the ferroportin 1 gene are responsible for type IV haemochromatosis.

In erythroid precursors, expression of TfR1, DMT1 and ferritin are reciprocally regulated through IRP1 and IRP2, which act on the IREs present in their RNAs. Thus, when erythropoiesis is stimulated and increased iron supply is needed, expression of TfR1 and DMT1 is increased, whereas synthesis of ferritin is halted. ${ }^{2}$ Erythroid cells also contain a DMT1 -IRE isoform, which is regulated by miR-Let-7d, a member of a new class of small non-coding single-stranded RNAs of $\sim 22$ nucleotides, the so-called microRNAs. MiR-Let-7d shows an opposite expression trend to DMT1 -IRE. When microRNA concentrations increase, the expression of DMT1 -IRE is inhibited and vice versa. With respect to DMT1 +IRE, the expression of the DMT1 -IRE isoform increases significantly more at the last stage of erythroid differentiation. ${ }^{32}$ In addition, there is evidence that EPO activates IRP-1, leading to upregulation of TfR1 expression in the erythroid precursors, which is maintained throughout the differentiation process. ${ }^{27}$ So far, several patients have been reported with DMT1 mutations causing microcytic hypochromic anaemia, due to decreased erythroid iron utilisation, but leading to increased liver iron stores. ${ }^{33}$

As stated above, senescent red blood cells are degraded by the proteolysis machinery of the phagolysosome in macrophages. The haem molecule reaches the cytosol, where it can act as a sensor molecule regulating the transcription of several genes, or be degraded by haem oxygenase 1 to release its iron. This iron will subsequently be stored in ferritin molecules for further use or exported back to the plasma by ferroportin 1. In macrophages, as well as duodenal enterocytes, ferroportin 1 is mostly found at the plasma membrane, but it is also present in intracellular vesicles after stimulation of its synthesis by intracellular iron.

A previous study carried out on primary cultures of bone marrow-derived macrophages had already proposed that erythrophagocytosis induces ferroportin 1, haem oxygenase 1 and ferritin mRNA expression by a haem-mediated pathway, followed by stimulation of ferroportin 1 and ferritin mRNA translation by an iron-dependent mechanism. ${ }^{34}$ More recently, Marro et $a l^{35}$ have shown that ferroportin 1 is transcriptionally coregulated with haem oxygenase 1 by haem. The protoporphyrin ring of haem is sufficient to increase ferroportin 1 gene transcriptional activity, while the iron released from the haem moiety controls ferroportin 1 translation involving the IRE in the 5' untranslated region. These findings suggest that haem controls macrophage iron recycling to ensure the coordinated degradation of haem by haem oxygenase 1 , iron storage and detoxification by ferritin, and iron export by ferroportin.

However, the mechanisms controlling the distribution of iron between the 'storage' compartment and the 'exportation' compartment have not been fully elucidated. It has been proposed that the net amount of ferroportin 1 present at the cell membrane, which is tuned to the erythroid iron needs by systemic signalling, facilitates iron mobilisation from the ferritin-associated iron stores. ${ }^{36}$ In this regard, EPO has been shown to reduce iron retention in macrophages by decreasing hepcidin and DMT1 expression and increasing ferroportin 1 expression. $^{26} 37$

On the other hand, besides these intrinsic regulations, the amount of ferroportin 1 at the plasma membrane is tightly controlled by systemic regulation relying on ferroportin 1 -hepcidin interaction. As for duodenal enterocytes, the binding of hepcidin to ferroportin 1 at the macrophage membrane causes its internalisation and degradation via a Janus kinase 2-clathrin-ubiquitin-mediated pathway. In addition, an independent lipid raft-mediated pathway for ferroportin internalisation and degradation has been recently described as a new route for hepcidin-mediated regulation of ferroportin 1 in macrophages. ${ }^{38}$ Nevertheless, it has been described that macrophages respond more acutely to a hepcidin challenge than the duodenal enterocytes, and this observation appears to be fully consistent with the central role of macrophages in maintaining body iron homoeostasis. ${ }^{39}$

As stated above, the liver is another main storage organ for iron. In iron overload, TfR1 is downregulated in hepatocytes. ${ }^{5}$ TfR2, which is highly expressed in human liver, lacks an IRE and thus is not reciprocally regulated in response to the concentration of plasma iron. Instead, TfR2 protein expression is regulated by transferrin saturation, and is upregulated in iron overload. In normal and iron-loaded conditions, expression of TfR2 exceeds that of TfR1, suggesting that TfR2 plays an important role in hepatic iron loading in haemochromatosis. ${ }^{1}$

\section{EFFECTS OF INFLAMMATION ON IRON HOMOEOSTASIS AND ERYTHROPOIESIS}

Anaemia is a common complication of chronic inflammatory diseases (eg, cancer, rheumatoid arthritis, inflammatory bowel diseases, congestive heart failure), as well as of sepsis and chronic renal failure. This anaemia may be the result of activation of the immune system by the underlying process, and certain immune and inflammatory cytokines including tumour necrosis factor alpha (TNF $\alpha$ ), interferon gamma (IFN $\gamma$ ) and interleukins (IL) 1 , 6, 8 and $10 .{ }^{40} 41$

As for anaemia of chronic disease (ACD), several pathophysiological mechanisms (cytokines) may be involved ${ }^{41}$ (figure 3 ):

1. Decreased red blood cell half-life due to dyserythropoiesis, red blood cell damage, and increased erythrophagocytosis (TNF $\alpha$ ).

2. Inadequate EPO responses for the degree of anaemia in most, but not all, patients, such as those with systemic-onset of juvenile chronic arthritis (IL-1 and TNF $\alpha$ ). ${ }^{42}$

3. Impaired responsiveness of erythroid cells to EPO (IFN $\gamma$, IL-1, TNF $\alpha$, hepcidin). ${ }^{43}$

4. Inhibited proliferation and differentiation of erythroid cells (IFN $\gamma$, IL-1, TNF $\alpha$, and $\alpha 1$-antitrypsin).

5. Pathological iron homoeostasis due to increased DMT1 (IFN $\gamma$ ) and TfR (IL-10) expression in macrophages, reduced ferroportin 1 expression (IFN $\gamma$ - and IL-6-induced high hepcidin concentrations) in enterocytes (inhibition of iron absorption) and macrophages (inhibition of iron recirculation), and increased ferritin synthesis (TNF $\alpha$, IL-1, IL-6, IL10) (increased iron storage)

Inflammatory cytokines such as IL-6 upregulate hepcidin transcription through activation of Janus kinases that phosphorylate Stat3, which become activated. ${ }^{44}$ Translocation of Stat 3 to the nucleus and its binding to the signal transducer 


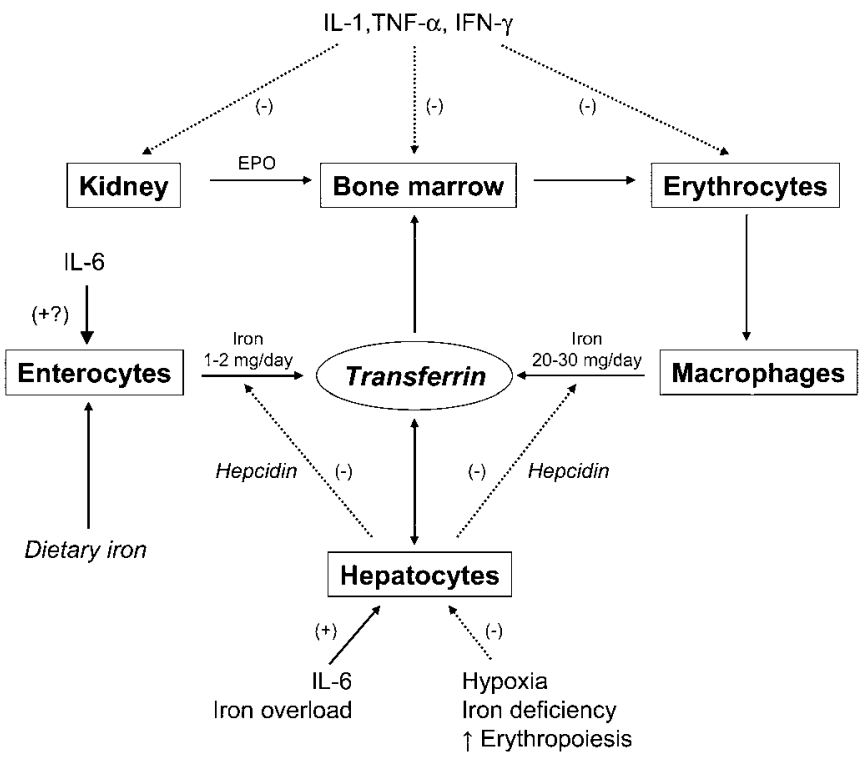

Figure 3 Effects of inflammation on erythropoiesis and iron homoeostasis in mammals (modified from Muñoz et al $\left.l^{2}\right) .(-)$, negative effect; $(+)$, positive effect; EPO; erythropoietin; IFN, interferon; IL, interleukin; TNF, tumour necrosis factor. See text for further details.

and activator of transcription (Stat) responsive element located in the proximal promoter of the hepcidin gene results in increased hepcidin release. This element appears to be regulated through the nearby BMP-responsive element via Smad activation, which is required for full promoter activity. A distal BMPresponsive element site is thought to interact with the Smad-Stat complex, which brings distal and proximal regions of the hepcidin promoter into physical contact. ${ }^{45}$ Thus, it seems that Smad signalling plays a key role in staging the appropriate response to inflammation. It has been further proposed that Stat3 activation itself, without inflammation, can regulate hepcidin concentrations (eg, patients with glycogen storage disease type $1 \mathrm{a}$ who have hepatic adenomas overexpressed hepcidin due to Stat3 activation) ${ }^{46}$ (figure 2 ).

Stress pathways signalling through the cellular endoplasmic reticulum unfolded protein response have also been found to induce hepcidin expression. The unfolded protein response has been implicated in the hepatic acute-phase response to lipopolysaccharide, IL- 6 and IL- $1 \beta$, suggesting that hepcidin gene expression may be regulated through an additional layer of endogenous control during inflammation ${ }^{47} 48$ (figure 2). All these lead to low serum iron and decreased transferrin saturation despite normal or high serum ferritin, through iron diversion to the RES (functional iron deficiency, FID), iron-restricted erythropoiesis and mild-to-moderate anaemia.

However, the pathophysiology of acute inflammation-related anaemia (eg, trauma, surgery) is somewhat different. In this setting, inflammatory responses are mediated mainly by IL- 6 and IL-8 with transient contribution from TNF $\alpha$ and IL- 1 in some visceral surgeries, such as gastrointestinal or cardiac procedures, whereas plasma IFN $\gamma$ concentrations are undetectable or within the normal range. ${ }^{49-51}$ Therefore, in most of these conditions, the two major mechanisms leading to anaemia are perioperative or traumatic blood loss and blunted erythropoiesis due to decreased iron availability, whereas EPO concentrations are normal or near normal. ${ }^{52}$

In the event of persisting decreased iron absorption and/or chronic blood loss, ACD may evolve to anaemia of chronic

\section{Take-home messages}

Total body iron is about $3500 \mathrm{mg}$ ( $50 \mathrm{mg} / \mathrm{kg}$ body weight), of which $65 \%$ is distributed in red blood cell haemoglobin (2300 mg).

- Absorption of dietary iron (1-2 mg/day) is tightly regulated, and just balanced against iron loss. Thus, internal turnover of iron is essential to meet the requirements of erythropoiesis (20-30 mg/day).

- Macrophages and liver are the main iron-storage sites (ferritin), but transferrin-bound iron $(3-4 \mathrm{mg})$ is the most important functional iron pool.

- Hepcidin, which modulates the expression of ferroportin, is the main iron-regulatory hormone of iron metabolism, and its synthesis is controlled by multiple signalling pathways (eg, inflammation, hypoxia, erythropoietin).

disease with true iron deficiency (ACD+ID). Interestingly, patients with $\mathrm{ACD}+\mathrm{ID}$ have significantly lower hepcidin concentrations than those with ACD, and are able to absorb some dietary iron from the gut and to mobilise some iron from macrophages. Thus, circulating hepcidin concentrations in ACD + ID seems to be more responsive to the erythropoietic demands for iron than to inflammation. ${ }^{53}$

Finally, it must be borne in mind that iron is not only required for erythropoiesis and oxidative metabolism. Cellular immune responses are also dependent on the presence of iron, and specific defects in cell-mediated immunity have been described in detail, even in mild iron deficiency, including the impaired proliferation and function of lymphocytes and natural killer cells, and a depressed neutrophil respiratory burst. ${ }^{54} 55$ For this reason, systemic inflammatory response episodes last longer in critically ill patients with FID, with prolonged stay in the intensive care unit and increased morbidity. ${ }^{56}$ The effectiveness of the administration of iron sucrose, alone or in combination with recombinant human $\mathrm{EPO}$, was assessed in a population of anaemic critically ill patients. ${ }^{57}$ Compared with those in the control group who only received folic acid, both treatments resulted in a trend to reduce the requirements for allogeneic blood transfusion (ABT). In addition, patients treated with iron sucrose experienced amelioration of the systemic inflammatory response, as assessed by C-reactive protein concentrations, and

\section{Interactive multiple choice questions}

- This JCP best practice article has an accompanying set of multiple choice questions (MCOs). To access the questions, click on BMJ Learning: Take this module on BMJ Learning from the content box at the top right and bottom left of the online article. For more information please go to: http://jcp. bmj.com/education Please note: the MCOs are hosted on BMJ Learning - the best available learning website for medical professionals from the BMJ Group. If prompted, subscribers must sign into JCP with their journal's username and password. All users must also complete a one-time registration on BMJ Learning and subsequently log in (with a BMJ Learning username and password) on every visit. 
a trend to lower mortality than those from the control group. These beneficial effects were not as evident in patients receiving iron sucrose plus recombinant human EPO, as blood marrow stimulation by recombinant human EPO may cause further FID.

Thus, iron deficiency or FID may lead not only to blunted erythropoiesis and chronic fatigue but also to an inappropriate immune response. On the other hand, the evidence that iron overload (or iron supplementation in iron-replete individuals) can promote both infectious and chronic inflammatory diseases is also clear. Thus, iron treatment in patients with active infectious diseases or ongoing bacteraemia should be avoided.

\section{Competing interests None.}

Provenance and peer review Commissioned; externally peer reviewed.

\section{REFERENCES}

1. Siah CW, Ombiga J, Adams LA, et al. Normal iron metabolism and the physiopathology of iron overload disorders. Clin Biochem Rev 2006;27:5-16.

2. Muñoz M, Villar I, García-Erce JA. An update on iron physiology. World J Gastroenterol 2009;15:4617-26

3. Andrews NC. Disorders of iron metabolism. N Eng/ J Med 1999;341:1986-95.

4. Fleming RE, Bacon BR. Orchestration of iron homeostasis. N Engl J Med 2005;352:1741-4.

5. Marignani M, Angeletti S, Bordi C, et al. Reversal of long-standing iron deficiency anaemia after eradication of Helicobacter pylori infection. Scand J Gastroenterol 1997;32:617-22.

6. Krishnamurthy $\mathbf{P}$, Xie T, Schuetz JD. The role of transporters in cellular heme and porphyrin homeostasis. Pharmacol Ther 2007;114:345-58.

7. Andrews NC. Understanding heme transport. N Engl J Med 2005;353:2508-9.

8. Zhang AS, Enns CA. Molecular mechanisms of normal iron homeostasis. Hematology Am Soc Hematol Educ Program 2009:207-14.

9. Crichton RR, Danielsson BG, Geisser P. Iron therapy with special emphasis on intravenous administration. Bremen: UNI-Med Verlag AG, 2008.

10. Andrews NC. Forging a field: the golden age of iron biology. Blood 2008:112:219-30.

11. Sheftel AD, Zhang A, Brown C, et al. Direct interorganellar transfer of iron from endosome to mitochondrion. Blood 2007;110:125-32.

12. Imlay JA, Chin SM, Linn S. Toxic DNA damage by hydrogen peroxide through the fenton reaction in vivo and in vitro. Science 1988;240:640-2.

13. Ramey G, Deschemin JC, Durel B, et al. Hepcidin targets ferroportin for degradation in hepatocytes. Haematologica 2010;95:501-4

14. Camaschella C. BMP6 orchestrates iron metabolism. Nat Genet 2009;41:386-8

15. Zhang AS, Gao J, Koeberl DD, et al. The role of hepatocyte hemojuvelin in the regulation of bone morphogenic protein-6 and hepcidin expression in vivo. J Biol Chem 2010;285:16416-23.

16. Lin L, Goldberg YP, Ganz T. Competitive regulation of hepcidin mRNA by soluble and cell-associated hemojuvelin. Blood 2005;106:2884-9.

17. Gao J, Chen J, Kramer M, et al. Interaction of the hereditary hemochromatosis protein HFE with transferrin receptor 2 is required for transferrin-induced hepcidin expression. Cell Metab 2009;9:217-27.

18. Schmidt PJ, Toran PT, Giannetti AM, et al. The transferrin receptor modulates Hfedependent regulation of hepcidin expression. Cell Metab 2008;7:205-14.

19. Pietrangelo A. Hereditary hemochromatosis: pathogenesis, diagnosis, and treatment. Gastroenterology 2010:139:393-408.

20. Lee PL, Beutler E. Regulation of hepcidin and iron-overload disease. Annu Rev Pathol Mech Dis 2009;4:489-515

21. Ryan JD, Ryan E, Fabre A, et al. Defective bone morphogenic protein signalling underlies hepcidin deficiency in HFE hereditary hemochromatosis. Hepatology 2010:52:1266-73.

22. Silvestri L, Pagani A, Nai A, et al. The serine protease matriptase-2 (TMPRSS6) inhibits hepcidin activation by cleaving membrane hemojuvelin. Cell Metab 2008:8:502-11.

23. Finberg KE, Heeney MM, Campagna DR, et al. Mutations in TMPRSS6 cause ironrefractory iron deficiency anemia (IRIDA). Nat Genet 2008;40:569-71.

24. Melis MA, Cau M, Congiu R, et al. A mutation in the TMPRSS6 gene, encoding a transmembrane serine protease that suppresses hepcidin production, in familial iron deficiency anemia refractory to oral iron. Haematologica 2008;93:1473-9.

25. Huang $\mathbf{H}$, Constante $\mathbf{M}$, Layoun $\mathrm{A}$, et al. Contribution of STAT3 and SMAD4 pathways to the regulation of hepcidin by opposing stimuli. Blood 2009;113:3593-9.

26. Ashby DR, Gale DP, Busbridge M, et al. Erythropoietin administration in humans causes a marked and prolonged reduction in circulating hepciin. Haematologica 2010:95:505-8

27. Weiss G, Houston $T$, Kastner $\mathrm{S}$, et al. Regulation of cellular iron metabolism by erythropoietin: activation of iron-regulatory protein and up-regulation of transferrin receptor expression in erythroid cells. Blood 1997:89:680-7.
28. Tanno T, Bhanu NV, Oneal PA, et al. High levels of GDF15 in thalassemia suppress expression of the iron regulatory protein hepcidin. Nat Med 2007;13:1096-101.

29. Tanno T, Porayette A, Orapan S, et al. Identification of TWSG1 as a second novel erythroid regulator of hepcidin expression in murine and human cells. Blood 2009;114:181-6

30. Peyssonnaux C, Zinkernagel AS, Schuepbach RA, et al. Regulation of iron homeostasis by the hypoxiainducible transcription factors (HIFs). J Clin Invest 2007; 117:1926-32.

31. Shah YM, Matsubara T, Ito S, et al. Intestinal hypoxia-inducible transcription factors are essential for iron absorption following iron deficiency. Cell Metab 2009:9:152-64.

32. Andolfo I, De Falco L, Asci R, et al. Regulation of Divalent Metal Transporter 1 (DMT1) non-IRE isoform by the microRNA Let-7d in erythroid cells. Haematologica 2010;95:1244-52.

33. Iolascon A, De Falco L, Beaumont C. Molecular basis of inherited microcytic anemia due to defects in iron acquisition or heme synthesis. Haematologica 2009:94:395-408.

34. Delaby C, Pilard N, Puy H, et al. Sequential regulation of ferroportin expression after erythrophagocytosis in murine macrophages: early mRNA induction by haem, followed by irondependent protein expression. Biochem $J$ 2008;411:123-31.

35. Marro S, Chiabrando D, Messana E, et al. Heme controls ferroportin1 (FPN1) transcription involving Bach1, Nrf2 and a MARE/ARE sequence motif at position -7007 of the FPN1 promoter. Haematologica 2010;95:1261-8.

36. De Domenico I, Vaughn MB, Li L, et al. Ferroportin-mediated mobilization of ferritin iron precedes ferritin degradation by the proteasome. Embo $J$ 2006;25:5396-404

37. Kong WN, Zhao SE, Duan XL, et al. Decreased DMTI and increased ferroportin I expression in the mechanisms of reduced iron retention in macrophages by erythropoietin in rats. J Cell Biochem 2008;104:629-41.

38. Auriac A, Willemetz A, Canonne-Hergaux F. Lipid raft-dependent endocytosis: a new route for hepcidin-mediated regulation of ferroportin in macrophages. Haematologica 2010:95:1269-77.

39. Chaston T, Chung B, Mascarenhas $M$, et al. Evidence for differential effects of hepcidin in macrophages and intestinal epithelial cells. Gut 2008;57:374-82.

40. Nowrousian MR, Kasper C, Oberhoff C, et al. Pathophysiology of cancer-related anemia. In: Smyth JF, Boogaerts MA, Ehmer BRM, eds. rhErythropoietin in cancer supportive treatment. New York: Marcel Dekker, 1996:13-34.

41. Weiss G, Goodnough LT. Anemia of chronic disease. N Engl J Med 2005;352:1011-23

42. Cazzola M, Ponchio L, de Benedetti F, et al. Defective iron supply for erytrhopoiesis and adequate endogenous erythropoietin production in anemia associated with systemic-onset juvenile chronic arthritis. Blood 1996;87:4824-3.

43. Raja KB, 0 Latunde-Dada G, Peters TJ. Role of interleukin- 6 in hypoxic regulation of intestinal iron absorption. Br J Haematol 2005;131:656-62.

44. Wrighting DM, Andrews NC. Interleukin-6 induces hepcidin expression through STAT3. Blood 2006;108:3204-9.

45. Wessling-Resnick M. Iron homeostasis and the inflammatory response. Annu Rev Nutr 2010;30:18.1-18.18

46. Weinstein DA, Roy CN, Fleming MD, et al. Inappropriate expression of hepcidin is associated with iron refractory anemia: implications for the anemia of chronic disease. Blood 2002;100:3776-81.

47. Zhang $\mathbf{K}$, Shen $\mathrm{X}, \mathrm{Wu}$ J, et al. Endoplasmic reticulum stress activates cleavage of CREBH to induce a systemic inflammatory response. Cell 2006;124:587-99.

48. Medzhitov R. Recognition of microorganisms and activation of the immune response. Nature 2007:449:819-26.

49. Jansson K, Redler B, Truedsson L, et al. Intraperitoneal cytokine response after major surgery: higher postoperative intraperitoneal versus systemic cytokine levels suggest the gastrointestinal tract as the major source of the postoperative inflammatory reaction. Am J Surg 2004;187:372-7. Related Articles.

50. Franke A, Lante W. Fackeldey $\mathrm{V}$, et al. Pro-inflammatory cytokines after different kinds of cardio-thoracic surgical procedures: is what we see what we know?. Eur $J$ Cardiothorac Surg 2005;28:569-75.

51. Muñoz M, García-Vallejo JJ, Sempere JM, et al. Acute phase response and cytokine release in patients undergoing lumbar spinal surgery: modulation by perioperative treatment with naproxen and famotidine. Eur Spine J 2004;13:367-73.

52. Van Iperen CE, Kraaijenahgen RJ, Biesma DH, et al. Iron metabolism and erythropoiesis after surgery. Br J Surg 1998;85;41-5.

53. Theurl I, Aigner $\mathrm{E}$, Theurl $\mathrm{M}$, et al. Regulation of iron homeostasis in anemia of chronic disease and iron deficiency anemia: diagnostic and therapeutic implications. Blood 2009;113:5277-86.

54. Oppenheimer SJ. Iron and its relation to immunity and infectious disease. J Nutr 2001;131:616S-35S.

55. Scrimshaw NS, SanGiovanni JP. Synergism of nutrition, infection, and immunity: an overview. Am J Clin Nutr 1997:66:464S-77S

56. Bellamy MC, Gednaey JA. Unrecognised iron deficiency in critical illness. Lancet 1998:352:1903.

57. Van Iperen CE, Gaillard CA, Kraaijenhagen RJ, et al. Response of erythropoiesis and iron metabolism to recombinant human erythropoietin in intensive care unit patients. Crit Care Med 2000:28:2773-8. 